\title{
Reference Vibration analysis of Piezoelectric Micromachined Modal Gyroscope
}

\author{
Cong Zhao, Xiao-Sheng Wu*, Guo-Bin Li, Wen-Yuan Chen, Wu Liu, Feng Cui, \\ Wei-Ping Zhang
}

(National Key Laboratory of Science and Technology on Micro/Nano Fabrication Laboratory, Shanghai Key Lab of Navigation and Location-based Services, Department of Micro-Nano Electronics, School of Electronic Information and Electrical Engineering, Shanghai Jiao Tong University, Shanghai 200240, China )

Keywords: piezoelectric, micro gyroscope, reference vibration, finite element

\begin{abstract}
Piezoelectric Micromachined Modal Gyroscope (PMMG) researched in the paper is a novel kind of angular rate sensor, which is based on high order resonant mode of a piezoelectric body as its reference vibration. Three methods, finite element method (FEM), scanning vibrometer and impedance analyzer, were applied to analyze the reference vibration. Reference resonant mode and the modal frequency and modal shape were obtained. Vibrating shape and resonant frequency were visualized and measured through scanning vibrometer equipment. Impedance analyzer was used in the equivalent circuit analysis. Reference vibrating frequencies obtained from mentioned methods are almost same, which are $343.500 \mathrm{kHz}, 346.094 \mathrm{kHz}$ and $347.316 \mathrm{kHz}$ respectively. The work of the paper provides the theoretical and experimental foundation for improving performance of PMMG.
\end{abstract}

\section{Introduction}

In conventional vibratory gyroscope, vibrating mass and its supporting springs are separated. Their reference vibration frequency is $1-20 \mathrm{kHz}$. For the piezoelectric micromachined modal gyroscope (PMMG), its vibrating mass and supporting springs are merged in one piezoelectric resonator as a whole. Reference vibration frequency of PMMG is $300-500 \mathrm{kHz}$, which is one to two orders higher than that of conventional vibratory gyroscope. Higher reference vibration frequency of the gyroscope will be good to reduce the mechanical Brownian noise and improve the gyroscope performance [2]. The device also shows reduced susceptibility to air damping [3]. Higher stiffness of PMMG is beneficial to resist shake and shock.

In this paper, working principle and reference mode of PMMG are detailed. Meanwhile modal and harmonic analysis are conducted through FEM. Then laser scanning vibrometer is utilized to visualize the vibrating shape and measure resonant frequency. At last, impedance of the gyroscope is tested by impedance analyzer. The test results are highly consistent with the simulation results, which verify the FEM analysis results and working principle of PMMG.

\section{Mechanism and working principle}

The structure of PMMG is showed in Fig.1, where L, W and H represent the length (x-axis), width (y-axis), height (z-axis, the direction of polarization P) of the piezoelectric vibrator. PMMG is composed of only prism-shaped bulk-PZT (Lead Zirconate Titanate: piezoelectric material) with driving electrodes (D+, D-), sensing electrodes (S1-S8) and reference electrodes (R1-R4) on its surfaces. When a special vibratory mode (reference resonance mode) is excited by an applied alternating voltage on driving electrodes and angular velocity is input, Coriolis force will be generated. The generated Coriolis force will induce differential voltage on sensing electrodes. The differential voltage processed by the circuit is used to detect the rotation rate inputted.

According to the analysis above, the shape of reference mode is the basis for generating Coriolis Effect. It is concluded that the reference resonance mode should have the following characteristics. (1) The movement of points in the piezoelectric block should be almost in one direction, $x$-axis or $y$-axis. 
(2) The moving direction of points should be perpendicular to the polarization direction of the piezoelectric block. (3) The moving direction of a point on one edge is the same as that of the corresponding point on the diagonal edge, and is opposite to that of the corresponding point on the adjacent edge. (4) Moving edges should be in the state of tension or compression. For the ideal reference mode, the points vibrate only in one direction, and there is no vibration in other two orthogonal directions. In fact, there is unwanted coupling displacement in the other two directions because of the coupling effect.

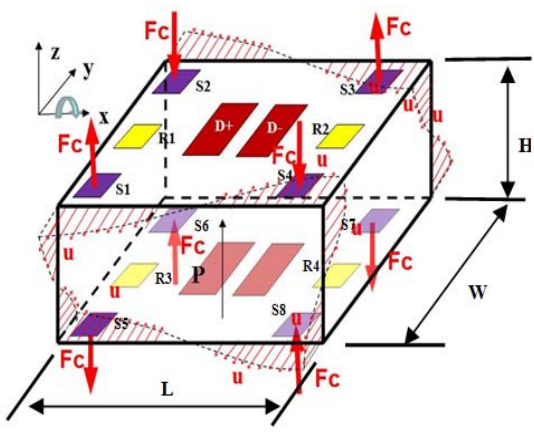

Fig.1. Working principle of PMMG

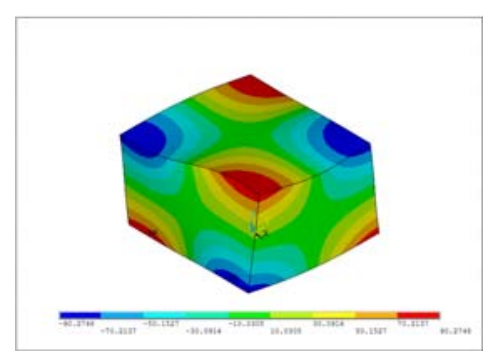

(a) y-axis displacement

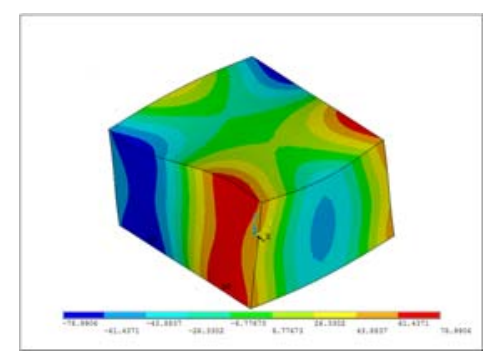

(b) z-axis displacement

Fig.2. The displacement contour ( $340.329 \mathrm{kHz}$ mode)

\section{FEM analysis and experimental verification of reference vibration of PMMG}

In this section, we utilize ANSYS (FEM analysis software) to simulate different vibration modes of PMMG within the specified frequency range. Modal and harmonic analysis are performed for confirming working resonance mode.

In the modal analysis, we choose piezoelectric element solid226 to simulate piezoelectric ceramic PZT-5H, which is selected as a substrate material for the gyroscope vibrator. PZT-5H material constants used in this paper are listed as follows: the dielectric constant ( $\varepsilon r): \varepsilon 11=762.5, \varepsilon 33=663.5$; material mass density $(\rho): 7500 \mathrm{~kg} / \mathrm{m}^{3}$; the piezoelectric constant $(\mathrm{e})$ : $\mathrm{e} 31=-5.20279 \mathrm{C} / \mathrm{m}^{2}$, e $33=$ $15.0804 \mathrm{C} / \mathrm{m}^{2}$, e16= $12.7179 \mathrm{C} / \mathrm{m}^{2}$; the elastic constant matrix $(\mathrm{c}): \mathrm{c} 11=13.9 \times 1010 \mathrm{~N} / \mathrm{m}^{2}$, $\mathrm{c} 12=7.8 \times 1010 \mathrm{~N} / \mathrm{m}^{2}, \mathrm{c} 13=7.4 \times 1010 \mathrm{~N} / \mathrm{m}^{2}, \mathrm{c} 33=11.5 \times 1010 \mathrm{~N} / \mathrm{m}^{2}, \mathrm{c} 44=2.6 \times 1010 \mathrm{~N} / \mathrm{m}^{2}$, $\mathrm{c} 66=3.1 \times 1010 \mathrm{~N} / \mathrm{m}^{2}$. The size of the model for piezoelectric block is selected as $5 \mathrm{~mm} \times 4 \mathrm{~mm} \times 3 \mathrm{~mm}$ and Block Lanczos algorithm is used to conduct modal analysis with the frequency scanned from 200 $\mathrm{kHz}$ to $400 \mathrm{kHz}$. Concerning the displacement in the y-axis direction, only the resonance mode at $340.329 \mathrm{kHz}$ can be satisfied. The displacement contour of $340.329 \mathrm{kHz}$ in the y-axis and z-axis direction is given in Fig.2.

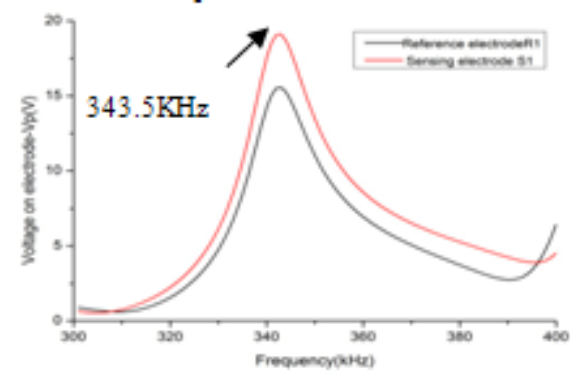

Fig. 3. Relation of the voltage output of R1and S1 to frequency 


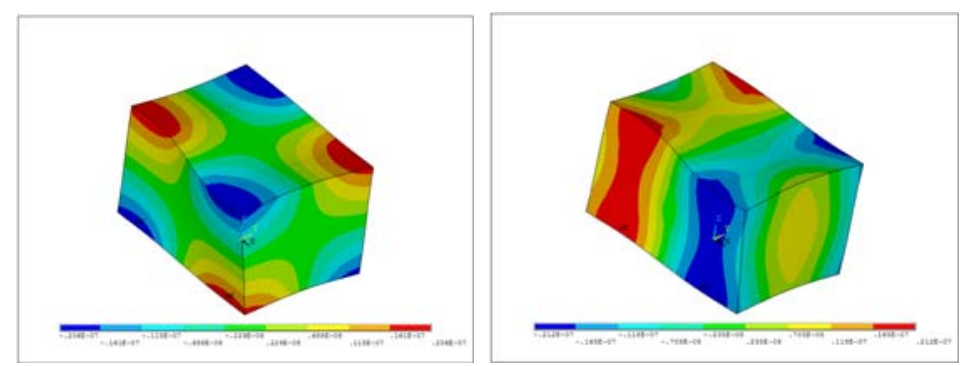

(a)y-axis displacement (b) z-axis displacement

Fig.4. The displacement contour of $343.5 \mathrm{kHz}$ under harmonic analysis

In order to validate the result of mode driving, we conducted the harmonic analysis using FEM. The frequency of the driving voltages should be the same as that of the reference mode, the amplitude is $5 \mathrm{~V}$, and the phase difference of the voltages on $\mathrm{D}+$ and $\mathrm{D}-$ is $180^{\circ}$. The damping constant of the piezoelectric material is introduced with the value of 0.02 . Fig. 3 gives the harmonic excitation analysis result, in which $\mathrm{x}$-coordinate refers to the frequency of the driving voltage and $\mathrm{y}$-coordinate refers to the piezoelectric voltage amplitude on reference electrode R1 and sensing electrode S1. The peak of both electrodes corresponding to the working resonance mode is in the position of around $343.5 \mathrm{kHz}$. The result of modal analysis is $340.329 \mathrm{kHz}$. The frequency difference of modal analysis and harmonic analysis is caused by the scanning frequency step in harmonic analysis.

Fig. 4 is the vibrating shape of piezoelectric block under the exciting frequency of $343.5 \mathrm{kHz}$. Comparing Fig.2 and 4, it is concluded that the exciting vibration of the piezoelectric block is the same as the vibrating shape of the working resonance mode. That is to say that through applying exciting voltage with the frequency of the working resonance mode on the driving electrodes, the vibration of the working resonance mode can be obtained.

\section{Laser scanning vibrometer test}

In order to verify the results of the finite element simulation and the practical working resonance parameters of PMMG, Polytec scanning vibrometer PSV-300 is utilized to observe vibrating shape and measure resonant frequency. Scanning vibrometer test, based on Doppler Effect, is an optical detection method and has characteristics of non-contact measurement, high resolution and fast response. Fig.5 is the gyroscope sample which has the size of the model used in FEM and is fabricated by MEMS processing technology.

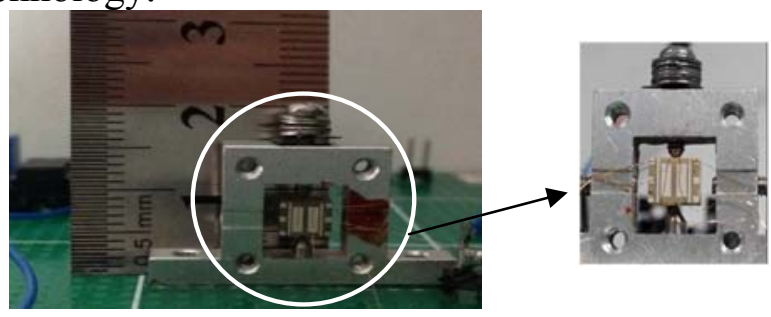

Fig. 5. Fabricated gyroscope device mounted on the supporting frame

Before the test, the excitation voltage of $2 \mathrm{Vp}$-p for reference vibration is applied at $\mathrm{D}+$ and $\mathrm{D}$-.The frequency is scanned from $300 \mathrm{kHz}$ to $400 \mathrm{kHz}$. Fig.6 gives the scanning results of resonance frequency in y direction and z direction. The peak frequencies of Fig.6 (a) and (b) are $346.094 \mathrm{kHz}$ and $346.406 \mathrm{kHz}$. Compare $346.094 \mathrm{kHz}$ with harmonic analysis result $(343.5 \mathrm{kHz})$, the relative error is $0.76 \%$.

Then xoz plane and xoy plane of the gyroscope are measured to obtain the vibration shape in $\mathrm{y}$-axis and z-axis direction under $346.094 \mathrm{kHz}$. Fig.7 gives the comparison of the mode shape of FEM simulation and scanning test, in which (a) and (c) refer to the y-axis displacement contour of xoz plane, (b) and (d) refer to z-axis displacement contour of xoy plane. 
From Fig.7 (a) and (c), (b) and (d), we can see that the simulated reference vibration of the block plane is the same as the measured vibrating shape of the working resonance mode, which verify the correctness of the finite element analysis mechanically.

Finally, we utilize Agilent 4294A precise impedance analyzer to test the impedance of PMMG. Relation of the impedance of PMMG to frequency is shown in Fig.8. From the figure we can see the two resonance frequencies are $293.726 \mathrm{kHz}$ and $347.316 \mathrm{kHz}$. The later frequency point meets the design requirements and has a relative error of $1.11 \%$ with the FEM harmonic result, which verify the reliability of finite element analysis electrically.

\section{Conclusion}

In this paper, reference vibration shape and working frequency of PMMG are analyzed in terms of simulation and experiments. Modal and harmonic analysis are performed to simulate the resonance modes for PZT5H prism through FEM. The simulated reference frequency is $343.5 \mathrm{kHz}$. Experimentally, reference shape and frequency are measured by scanning laser vibrometer and impedance analyzer and the results are $346.094 \mathrm{kHz}, 347.316 \mathrm{kHz}$. Comparing these results with simulation results, the relative errors are $1.0 \%$ and $1.4 \%$ respectively, which verifies the reliability of FEM experimentally. The research of this paper provides a theoretical and experimental foundation for the following measuring circuit.

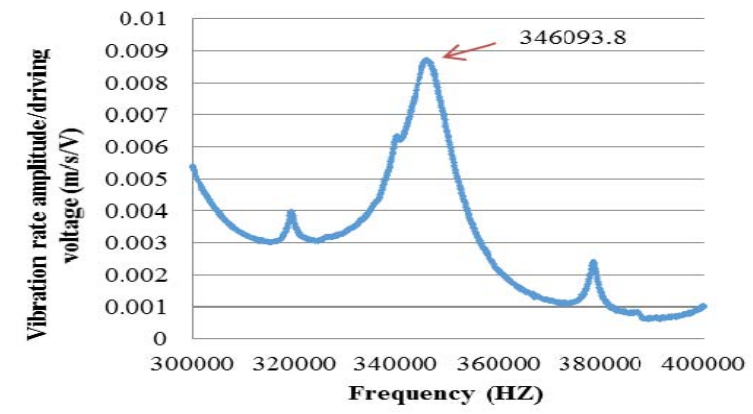

(a) scanning results in y direction

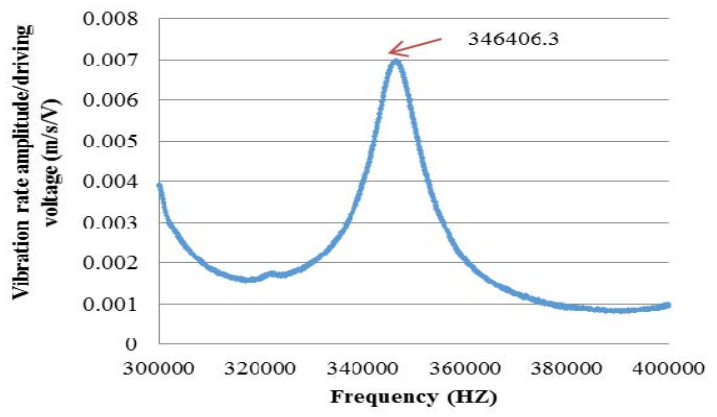

(b) scanning results in $\mathrm{z}$ direction

Fig.6. Scanning results of resonance frequency

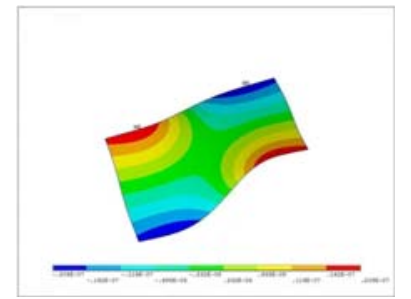

(a) y-axis displacement (b) z-axis displacement

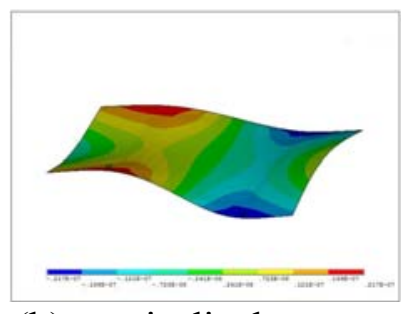

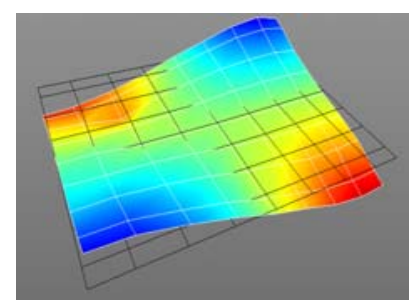

(c) y-axis displacement

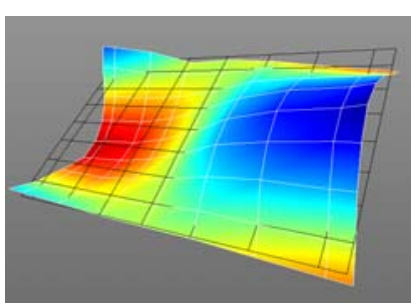

(d) z-axis displacement

Fig. 7. Comparison of the reference mode of FEM simulation and scanning test

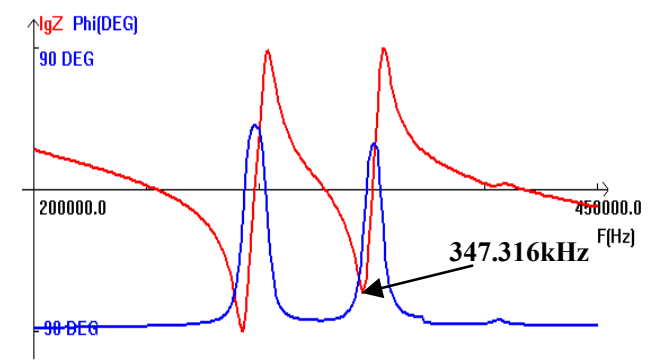

Fig. 8. Impedance curve of piezoelectric gyroscope 


\section{Acknowledgements}

This work was sponsored by the Shanghai Pujiang Program (14PJD022) and Supporting Project of Education Department of China (625010117), Corresponding author: xswu@sjtu.edu.cn

\section{References}

[1] K. Maenaka, H. Kohara, M. Nishimura, T. Fujita, and Y. Takayama, Novel solid micro-gyroscope, Proceedings of the 19th IEEE Conference on MEMS, Istanbul, Turkey, January2006, pp. 634-637

[2] Sharma A, Zaman M, Ayazi F, (2007) A 104 -dB dynamic range transimpedance -based CMOS ASIC for tuning fork microgyroscopes. IEEE J. Solid-State Circuits, 42: (8) pp1790 -1802

[3] H. Johari, J. Shah and F. Ayazi, High frequency xyz-axis single-disk silicon gyroscope, Proceedings of the 21st IEEE Conference on MEMS, Kobe, Tucson, AZ, USA, January2008, pp.856 - 859 